\title{
Philosophiques
}

\section{André Morazain et Salvatore Pucella, Éthique et politique. Des valeurs personnelles à l'engagement social. Montréal, Éditions du Renouveau Pédagogique Inc., 1988, 130 p.}

\section{Martin Gagnon}

Volume 16, numéro 1, printemps 1989

URI : https://id.erudit.org/iderudit/027073ar

DOI : https://doi.org/10.7202/027073ar

Aller au sommaire du numéro

Éditeur(s)

Société de philosophie du Québec

ISSN

0316-2923 (imprimé)

1492-1391 (numérique)

Découvrir la revue

Citer ce compte rendu

Gagnon, M. (1989). Compte rendu de [André Morazain et Salvatore Pucella, Éthique et politique. Des valeurs personnelles à l'engagement social. Montréal, Éditions du Renouveau Pédagogique Inc., 1988, 130 p.] Philosophiques, 16(1), 206-208. https://doi.org/10.7202/027073ar d'utilisation que vous pouvez consulter en ligne.

https://apropos.erudit.org/fr/usagers/politique-dutilisation/ 
ANDRÉ MORAZAIN et SALVATORE PUCELLA, Éthique et politique. Des valeurs personnelles à l'engagement social. Montréal, Éditions du Renouveau Pédagogique Inc., 1988, 130 p.

$$
\text { par Martin Gagnon }
$$

Principalement conçu pour les professeurs et les étudiants du cours de philosophie 401 de niveau collégial, ce manuel se veut tout d'abord une introduction simple et aussi nuancée que possible à l'approche philosophique de l'éthique et du politique. C'est donc dans cette optique d'introduction et de présentation qu'il se doit d'être lu.

Il en va de la signification du «et» dans «éthique et politique»: refusant la voie traditionnelle d'une distinction tranchée entre ces deux ordres de réflexion, Morazain et Pucella entendent bien plutôt démontrer que cette distinction est "dans une très large mesure artificielle et stérile» (p. XI) et que toute interrogation menée sur le plan des valeurs personnelles doit nécessairement déboucher sur une réflexion tenant compte des modalités de notre engagement social, d'où le sous-titre de l'ouvrage. 
Dans la première partie consacrée à l'étude des valeurs individuelles, les auteurs tentent de dégager la spécificité de la réflexion éthique en partant du dilemme moral. Étant donné qu'un tel dilemme présuppose toujours un conflit de valeurs, il appartient dès lors à l'éthique de nous aider à répondre à la question: "Que dois-je faire?» en montrant «comment nous pouvons déterminer le choix le plus raisonnable et le plus justifiable» (p. 8). Une telle justification n'étant valable qu'à partir d'une démarche rationnelle, l'éthique n'a pas à considérer les «arguments » provenant de sources plus «subjectives » comme la religion, l'opinion publique, les sentiments, etc... Ceci établi, il s'ensuit que toute tentative visant à mettre sur pied une théorie morale devra respecter deux exigences rationnelles : d'un part, elle devra identifier une valeur fondamentale (qu'aucune autre valeur ne serait donc susceptible de confronter) et d'autre parr, il lui faudra s'assurer de la portée universelle de cette valeur. Les auteurs terminent cette première partie en examinant deux théories morales qui semblent répondre à cette double exigence, soit le personnalisme et l'utilitarisme.

Dans la seconde partie de l'ouvrage, Morazain et Pucella se penchent sur "le cadre politique dans lequel s'effectue les choix des individus » (p. 47). S'il est vrai que les valeurs individuelles de chacun doivent beaucoup à la société qui favorise l'éclosion et la véhiculation de ces valeurs, il convient alors d'examiner de plus près les principales conceptions du pouvoir qui déterminent les buts que peuvent viser les membres appartenant à une même société. C'est ce que font les auteurs à partir des trois grandes conceptions du politique que représentent l'autoritarisme, l'anarchisme et la démocratie.

Enfin, c'est en cheminant des conceptions du pouvoir aux orientations idéologiques qu'elles impliquent que la réflexion portant sur l'essence du politique rencontre une question cruciale: "L'objectif de l'État est-il de garantir la liberté individuelle ou bien plutôt de promouvoir l'égalité sociale? » (p. 57). Parce qu'ils ont répondu différemment à cette question, libéralisme et socialisme se partagent actuellement le monde, chacun misant sur les valeurs collectives qu'il juge les plus fondamentales, soit la liberté et la sécurité dans le premier cas, égalité et justice dans le second. Mais le fait que dans certains cas les systèmes socialisant soient naturellement forcés de se « libéraliser » et que les démocraties soient parfois obligées de recourir à des tactiques «socialisantes» montre bien que chaque système comporte des limites : une telle “ ironie » idéologique manifeste le caractère non absolu des valeurs collectives que revendiquent ces deux orientations idéologiques.

Or, cette complémentarité du libéralisme et du socialisme qui se laisse surprendre sur le plan politique, les auteurs l'avaient déjà aperçue au niveau de la réflexion éthique portant sur les fondements du personnalisme et de l'utilitarisme en suggérant que si le personnalisme, avec l'insistance qu'il met sur le respect de la personne, peut parfois dépasser les apories de l'utilitarisme, il est vrai aussi que l'utilitarisme, qui mise sur l'accroissement du plaisir et la diminution de la douleur, peut parfois pallier aux culs-de-sac rencontrés par le personnalisme. 
Conscients de ces exigences éthiques, les auteurs croient que «par-delà la liberté et l'égalité », la réflexion devrait envisager une "troisième voie " grâce à laquelle le politique serait en mesure de tenir compte des exigences propres au personnalisme et à l'utilitarisme plutôt que de jouer l'un contre l'autre lorsqu'il s'agit de déterminer les valeurs qui devraient servir de fondements à la vie sociale: "Il nous semble que nous sommes confrontés depuis trop longremps à un faux dilemme : un pouvoir par le peuple (liberté) ou un pouvoir pour le peuple (égalité). Ce qu'il faut, c'est un pouvoir qui soit à la fois l'un et l'autre» (p. 116).

Ceci dit, les auteurs ont été soucieux, tout au long de l'ouvrage, de maintenir un certain équilibre entre l'argumentation théorique et la réflexion à partir des cas pratiques le plus souvent puisés dans la vie quotidienne ; les exemples sont la plupart du temps choisis avec soin et, dans certains cas, ne manquent pas d'imagination. De cette manière il est manifeste que le lecteur (c'est-à-dire l'étudiant) non seulement a la possibilité de se familiariser sur le plan concret avec les notions à l'étude, mais de plus, il est invité à clarifier ses prises de position personnelles sur le terrain d'une argumentation rationnelle qui ne se contente pas d'affirmer « moi je pense que... » mais qui se doit aussi de justifier ses affirmations. Nul doute que ce soit là la grande qualité pédagogique de cet ouvrage qui, par-delà ses propres conclusions, constitue une initiation sérieuse à l'approche philosophique des problèmes - souvent très délicats - de l'éthique et du politique.

Pour ce qui regarde les options théoriques de l'ouvrage, on aurait pu croire de prime abord - ne fut-ce que par un survol rapide de la table des matières - que Morazain et Pucella cédaient d'entrée de jeu à un vieux réflexe qui consiste à réduire l'éthique au politique. En fait, il n'en est rien car en dépit de la répugnance qu'ils éprouvent à traiter l'éthique et le politique comme deux ordres de choses séparés, il n'en demeure pas moins que la «troisième voie» qu'ils préconisent - le tiers exclu du libéralisme et du socialisme - est motivée par les requêtes fondamentales de l'éthique ellemême. Ceci donne à penser que si l'éthique et le politique doivent se concerner mutuellement, ce n'est pas afin que le politique puisse mieux «gober" l'éthique mais bien plutôt pour que l'appareillage critique de l'éthique puisse au besoin orienter ou rectifier les projets du politique.

"On peut donc conclure que le politique (...) n'est qu'un sous-ensemble de l'éthique» (p. 112). Conclusion que nous voudrions accepter mais que refuseraient probablement toutes ces victimes de la "haine de l'autre homme » si elles pouvaient encore crier.

Département de philosophie

Université de Montréal 